\title{
Definite visualized communication of a Baker's cyst with knee joint space and iatrogenic air content in rheumatoid arthritis patient
}

\author{
Hong Ki Min ${ }^{1}$, Se Hee Kim, Sung Gyu Moon², Hae-Rim Kim³, and Sang-Heon Lee ${ }^{3}$
}

${ }^{1}$ Division of Rheumatology, Department of Internal Medicine, Konkuk University Medical Center, Seoul; ${ }^{2}$ Department of Radiology, ${ }^{3}$ Division of Rheumatology, Department of Internal Medicine, Konkuk University School of Medicine, Seoul, Korea

Received: March 15, 2019

Revised : April 10, 2019

Accepted: April 10, 2019

\section{Correspondence to}

Sang-Heon Lee, M.D.

Tel: +82-2-2030-7541

Fax: +82-2-2030-7748

E-mail: shlee@kuh.ac.kr

https://orcid.org/oooo-ooo1-

7925-0907
A 77-year-old man with longstanding rheumatoid arthritis was hospitalized because of gastric ulcer bleeding. $\mathrm{He}$ was referred to a rheumatologist due to a large Baker's cyst in the right knee joint, which was incidentally found in computed tomography to rule out deep vein thrombosis. Fluid from Baker's cyst showed increased white blood cell count $(39,200$ cells $/ \mu \mathrm{L})$ with predominant neutrophils (88\%). Erythrocyte sedimentation rate (ESR) $(>120$ $\mathrm{mm} / \mathrm{hr}$ ) and high sensitivity C-reactive protein (hsCRP; $8.59 \mathrm{mg} / \mathrm{dL}$ ) levels were significantly elevated. Magnetic resonance imaging (MRI) revealed definite communication between Baker's cyst and knee joint space on T2-weighted fat-suppression axial (Fig. 1A) and T1-weighted magnetization prepared rapid acquisition gradient-echo axial
(Fig. 1B) images (white arrow) and on T2-weighted sagittal image (Fig. 1C; black arrow). Air bubbles (black arrow) were noted in the non-dependent area of Baker's cyst on T2-weighted sagittal image (Fig. 1D). Due to combined air content and elevated acute phase reactants, we could not rule out infection of Baker's cyst. After 7 days of intravenous antibiotic treatment, ESR and hsCRP levels did not improve; bacterial culture was negative. After systemic glucocorticoid administration, ESR/ hsCRP levels decreased to $72 \mathrm{~mm} / \mathrm{hr}$ and $0.56 \mathrm{mg} / \mathrm{dL}$, respectively. Treatment decision was difficult since the etiology of co-existing air in Baker's cyst was confusing (infection vs. iatrogenic). After excluding infection, we concluded that the air was from previous intra-articular injection (4 months
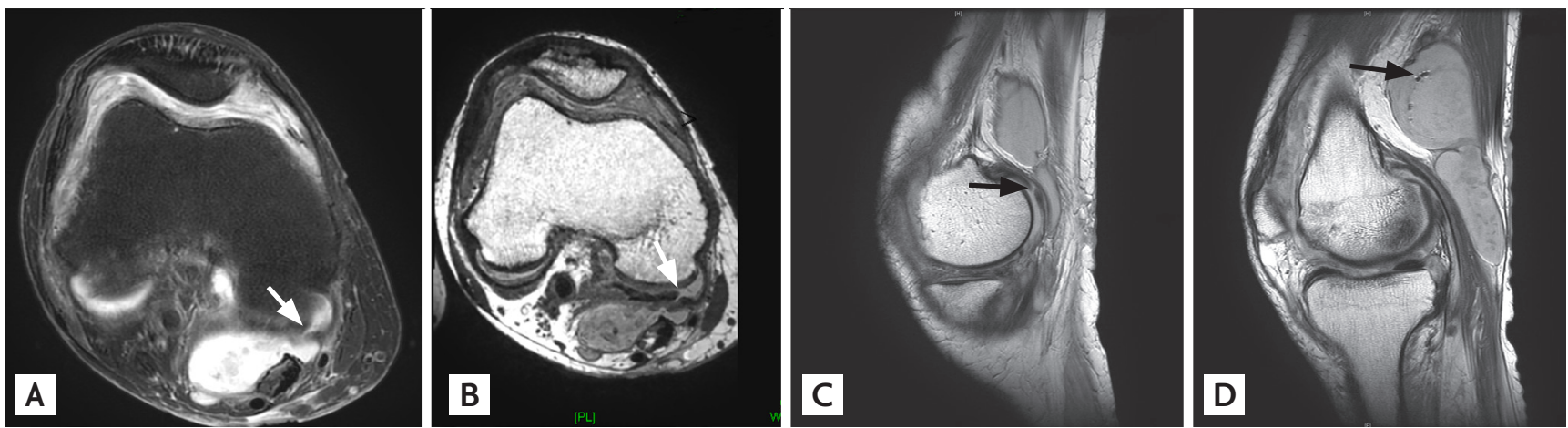

Figure 1. (A) The communication between Baker's cyst and knee joint is visualized (white arrow) in T2-weighted fat-suppression axial view, and (B) T1-weighted magnetization prepared rapid acquisition gradient-echo axial view. (C) The communication is pointed with black arrow in T2-weighted sagittal view. (D) Iatrogenic air bubbles are observed in Baker's cyst (black arrow). 
prior to admission). Baker's cyst is posterior synovial sac of knee joint, and located between the medial head of the gastrocnemius and semimembranosus tendons. Although autopsy and arthroscopic findings demonstrated communication between Baker's cyst and knee joint space, our case showing such communication on MRI is rare. Furthermore, iatrogenic air of Baker's cyst by joint injection has not been reported. These clinical images demonstrate excellent visualization of communication between a Baker's cyst and knee joint space with abnormal air content.

Informed consent was waived by the board.

\section{Conflict of interest}

No potential conflict of interest relevant to this article was reported. 